\title{
Acute on chronic liver failure: causative factors and comparison of prognostic scores
}

\begin{abstract}
Background: Acute-on-chronic Liver failure (ACLF) is a distinct entity, different from patients with cirrhosis and de compensation. However, despite being a devastating syndrome with inordinately high mortality, the precipitating factors or prognostic scoring systems remain ill defined. We studied the precipitating factors, role of various factors on outcome and compared prognostic scores in predicting short term (7days) outcome. Methods: 100 patients with ACLF were enrolled. All patients were worked up for possible etiologies of underlying CLD \& acute precipitating events \& were classified in various categories of ACLF accordingly. APACHE II, SOFA, CTP \& MELD scores were compared in terms of AUROC values for predicting short term mortality.

Results: The etiology of underlying cirrhosis was alcohol in majority of patients $(59 \%)$ with alcoholic hepatitis being the most common acute precipitating event, occurring in $43 \%$ of patients. The short term mortality was $55 \%$. There was a significant difference in the values of Mean arterial pressure $(p=0.044)$, Heart rate $(p=0.042)$, Platelet count $(p=0.001)$, S. Bilirubin $(p=<0.001)$, S. Sodium $(p=<0.001)$, S. Creatinine $(p=0.076)$, S. Albumin $(\mathrm{p}=<0.001) \&$ INR $(\mathrm{p}=<0.001)$ of survivors \& Non survivors. The short term mortality was comparatively higher in patients with SBP $(93 \%, p=0.001) \&$ UGI bleed $(100 \%, \mathrm{p}=0.003)$ as their precipitating causes while it was comparatively lower in patients with Acute viral hepatitis \& Hepatitis B virus flare/Autoimmune hepatitis flare $(13 \%, \mathrm{p}=<0.001)$. Overall APACHE II had a higher AUROC than SOFA, MELD, CTP ( 0.938 vs 0.782 for SOFA vs 0.780 for MELD and 0.669 for CTP).
\end{abstract}

Conclusion: APACHE II scoring system is superior to other prognostic scores in predicting short term mortality.

Keywords: cirrhosis, precipitating factor, apache ii, alcoholic hepatitis
Volume I Issue 3 - 2016

Rujul Jain, Manisha Bais T

Department of Medicine,Vardhman Mahavir Medical College \& Safdarjung Hospital, India

Correspondence: Rujul Jain, Department of Medicine, Vardhman Mahavir Medical College \& Safdarjung Hospital, New Delhi, India, Post Code I 10029, Tel +995873 I97I, Email rujulI0II@gmail.com

Received: November 15, 2015 | Published: January 09, 2016
Abbreviations: ACLF, acute-on-chronic liver failure; ROC, receiver operating characteristic curve; AUROC, area under the roc curve; SBP, spontaneous bacterial peritonitis

\section{Introduction}

Acute on chronic liver failure (ACLF) is defined as an acute hepatic insult manifesting as jaundice and coagulopathy, complicated within 4weeks by ascites and/or encephalopathy in a patient with previously diagnosed or undiagnosed chronic liver disease. ${ }^{1}$ ACLF is a distinct entity, different from patients with cirrhosis and de compensation in two key elements. First the development of liver failure and end organ dysfunction in ACLF is much faster. Second (and may be of more importance), in ACLF, there is still a chance of recovery of liver function. Patients may revert back to the basic clinical status if the acute event and clinical consequences recover. ${ }^{2}$

Patients with ACLF have a high short-term mortality. The mortality rate is directly proportional to the number of organ failures observed. ${ }^{3}$ The treatment modalities are limited in patients with ACLF, therefore it is important to make prognosis for these patients. Assessment of prognostic criteria of ACLF will allow us to incorporate it as a possible new indicator of high urgency allocation of patients to ICU and also help in determining various other treatment protocols. Hence we did this study to evaluate the performance of various prognostic scores in prediction of short term mortality in patients with ACLF.

\section{Methods}

\section{Definition and inclusion criteria}

The study included 100 consecutive patients with ACLF admitted in the Medicine Wards in Vardhman Mahavir Medical College \& Safdarjung Hospital, New Delhi from Nov 2013 to Dec 2014. Written and informed consent was taken from all the subjects participating in the study. Ethical clearance was taken from ethical committee of the institute before conducting the study.

ACLF was diagnosed according to the Asian-Pacific Association for the study of the Liver (APASL) criteria. Acute insult was defined as serum bilirubin $>5 \mathrm{mg} / \mathrm{dl}$ and liver failure as $\mathrm{INR} \geq 1.5$ or pro thrombin activity $<40 \%$, complicated within 4 weeks by ascites and/or encephalopathy occurring in a patient with known or unknown CLD. ${ }^{1}$

CLD was defined as either the presence of cirrhosis or chronic hepatitis of any etiology. Cirrhosis was defined as diffuse process in the liver characterized by development of extensive fibrosis and replacement of normal liver architecture by structurally abnormal nodules of fibrotic tissue. ${ }^{4}$ Chronic hepatitis B was defined as ongoing injury to the cells of liver with persistence of HBsAg in serum for at least 6months. ${ }^{4}$ Diagnosis of Cirrhosis was based on clinical findings, biochemical findings (low serum albumin, AST: ALT ratio $>1$ ), imaging findings (USG findings of heterogeneous echotexture of liver with irregular outline, altered liver size depending on the etiology, portosystemic collaterals). 
All patients were worked up thoroughly for the possible etiologies of their underlying CLD and acute precipitating events, and were then classified in the various categories of ACLF accordingly. Based on the type of acute insult, patients were divided into three types: Type I (Infectious etiology), Type II (Non infectious etiology), Type III (Unknown hepatotoxic etiology). ${ }^{1}$ They were also divided into Type I (non-hepatic injury) and Type II (hepatic injury) according to whether hepatic or non hepatic cause was the acute precipitating event. SBP, various infections (like LRTI, UTI, Cellulites etc.) leading to sepsis/ MODS, acute variceal bleed, surgical interventions were classified as non hepatic insults precipitating ACLF. In the hepatic causes, acute viral hepatitis, drugs/toxins, alcoholic hepatitis, HBV flare were considered. ${ }^{5}$ Sepsis was considered as acute precipitating event only after common hepatic acute events including acute viral hepatitis, viral or autoimmune hepatitis flare and exposure to drugs and toxins were excluded by appropriate history and investigations.

\section{Clinical manifestations and prognostic scores}

History included asking the patients for Jaundice, abdominal distension, drowsiness/altered sensorium, fever, haematemesis or malena, alcohol consumption and other related symptoms. A detailed physical examination was also done. All routine blood investigations including those required for the calculation of various prognostic scores were obtained. All patients were evaluated for various prognostic scores after all the required parameters were available within 48hours of the patient's admission including APACHE II, SOFA, CTP and MELD, as per the standard definitions. All patients were followed up during the hospital stay. Short term mortality was defined as patients in hospital death and death within 1 month after leaving the hospital.

\section{Statistical analysis}

The data was expressed as median $(25-75 \%$ IQR). To study the causative factors and their outcome on the mortality comparisons were made between survivors and non survivors. Chi square test (for qualitative variables) and student $t$ test (for quantitative variables) were used. The Mann-Whitney U-test was used to compare the prognostic scores between the survivors and non-survivors. The performance of prognostic scores on the prediction of short term mortality was assessed by the receiver operating characteristic (ROC) curve. The area under the ROC curve (AUROC) was calculated for APACHE-II, SOFA, CTP and MELD scores in all patients. Sensitivity, specificity, positive predictive value and negative predictive value for the prediction of the short term mortality were determined. Measurement of agreement was based on kappa statistics. Statistical analysis was performed using SPSS version 21.0. $\mathrm{p}$ value $<0.05$ was taken as level of statistical significance.

\section{Results}

\section{Baseline clinical and biochemical characteristics}

Out of the 100 patients studied, the mean age was 52.37years ranging from $35-65$ years and the median age was 53years. 80 patients were males and 20 were females. Jaundice was present in all the cases and ascites with encephalopathy was the clinical presentation in $64 \%$ of the cases (Table 1)

\section{Etiology of CLD and acute insults}

The etiology of underlying cirrhosis was alcohol in 59(59\%), alcohol plus virus (HBV or HCV) in $6(6 \%), \mathrm{HBV}$ in $12(12 \%), \mathrm{HCV}$ in $9(9 \%)$, AIH in 3(3\%), Wilson's in $2(2 \%)$, NASH/cryptogenic in $9(9 \%)$ of patients (Table 1). Alcoholic hepatitis was the overall most common acute precipitating event followed by Spontaneous bacterial peritonitis (SBP) (Table 2).

Table I Baseline characteristics, etiology of liver cirrhosis and clinical presentations in patients with acute-on-chronic liver failure $(\mathrm{N}=100)$

\begin{tabular}{ll}
\hline Baseline characteristics & \\
\hline Age, (years median [25-75\% IQR]) & $53(35-65)$ \\
Sex, $n$ (M : F) & $80: 20: 00$ \\
Etiology of cirrhosis $(\mathrm{n})$ & 59 \\
Alcohol & 6 \\
Alcohol plus virus infection (HBV/HCV) & 12 \\
HBV & 9 \\
HCV & 3 \\
AlH & 9 \\
NASH/cryptogenic & 2 \\
Wilson's disease & \\
Clinical presentation (n) & $100(100 \%)$ \\
Jaundice & $95(95 \%)$ \\
Ascites & $64(64 \%)$ \\
Encephalopathy alone & $26(26 \%)$ \\
Fever &
\end{tabular}

Table 2 Etiology of acute events

\begin{tabular}{ll}
\hline Etiology & Patients(n) \\
\hline Infectious etiology & 41 \\
Hepatotropic viruses & 14 \\
Reactivation of Hep B or Hep C & 2 \\
Other infectious agents afflicting the liver & 25 \\
Non infectious etiology & 58 \\
Alcohol : active drinking within last 4 weeks & 43 \\
Hepatotoxic drugs & 2 \\
Flare of AlH or Wison's & 3 \\
Surgical intervention & 0 \\
Variceal bleed & 10 \\
Unknown hepatotoxic etiology & 1 \\
\hline
\end{tabular}

\section{Short-term mortality}

Out of the 100 patients, 48 patients died during the hospital stay and 7 patients left the hospital against medical advice with a very sick status and were confirmed to have died via telephone contact with the family. Hence the overall short-term mortality in our study came out to be $55 \%$.

\section{Comparison of prognostic scores and other factors}

There was a significant difference in the values of MAP $(\mathrm{p}=0.044)$, Heart rate $(\mathrm{p}=0.042)$, Platelet count $(\mathrm{p}=<0.001)$, S. bil $(\mathrm{p}=<0.001), \mathrm{S}$. Sodium ( $p=<0.001)$, S. Creatinine $(p=0.076)$, S. Albumin $(p=<0.001)$ 
and INR $(\mathrm{p}=<0.001)$ of survivors and non-survivors (Table 3a) (Table $3 b)$.

Table 3a Etiology and types of ACLF

\begin{tabular}{ll}
\hline Etiology and type of ACLF & Patients(n) \\
\hline Type I (non hepatic insult) & 35 \\
SBP & 15 \\
Cellulitis & 2 \\
UTI & 3 \\
LRTI & 3 \\
Sepsis/other sites of infection & 2 \\
UGI bleed & 10 \\
Post surgery decompensation & 0 \\
Type II (hepatic insult) & 64 \\
Type II A & \\
Acute viral hepatitis & 14 \\
Type II B & \\
Drug induced & 2 \\
Type II C & \\
Alcoholic hepatitis & \\
AlH flare & 33 \\
HBV flare & 2 \\
Unknown etiology & 1 \\
\hline
\end{tabular}

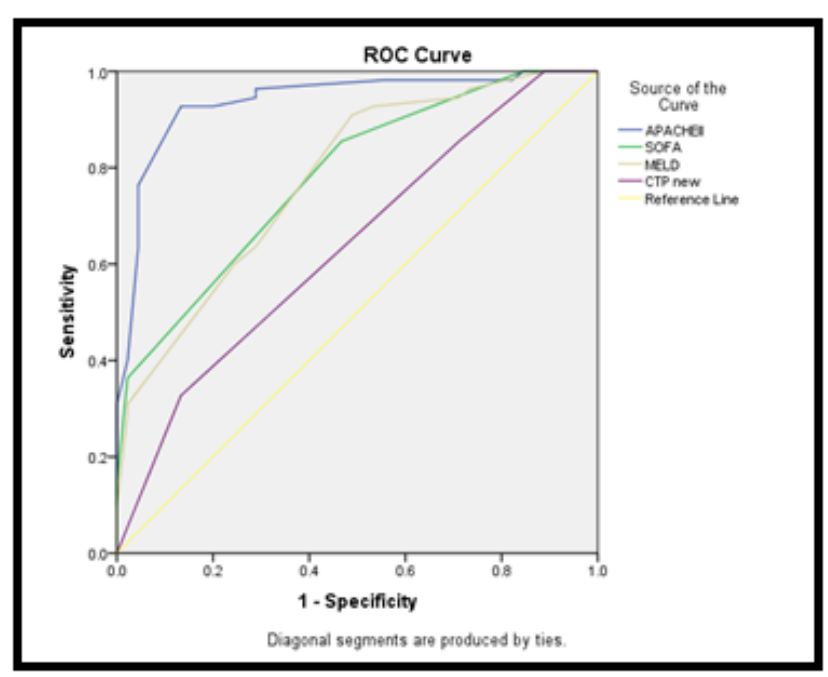

Figure I Comparison of various prognostic scores.

The short-term mortality is high in ACLF with the mortality comparatively higher in patients with UGI bleed, SBP and various other causes of sepsis/infection as the cause of precipitating event. All the patients with UGI bleed in our study presented with low MAP, hematocrit and required urgent packed cell transfusions. Patients with ACLF who died had significantly higher CTP, MELD, APACHE II and SOFA scores than survivors. $(\mathrm{p}=0.001)$. APACHE II had a higher AUROC than SOFA, MELD, CTP (0.938 vs 0.782 for SOFA, 0.780 for MELD and 0.669 for CTP). This shows the superiority of APACHE II in relation to other scores for predicting short-term mortality (Table 4).

Table 3b Comparison of various biochemical \& hemodynamic factors between survivors and non-survivors: [data represented in median (Inter quartile range)]

\begin{tabular}{llll}
\hline Variables & Non survivors[n=55] & Survivors[n=45] & P-value \\
\hline MAP $(\mathrm{mmHg})$ & $70(65.0-70.0)$ & $83(80-85)$ & 0.044 \\
HR $(/ \mathrm{min})$ & $98(88.0-110.0)$ & $84(80-90)$ & 0.042 \\
RR $(/ \mathrm{min})$ & $24(24.0-26.0)$ & $18(16-20)$ & 0.112 \\
Hct & $35.8(28.9-39.2)$ & $39(34.7-42.8)$ & 0.124 \\
TLC & $9700(7800-12900)$ & $9400(7730-10250)$ & 0.128 \\
Platelet count $\left(\times 10^{3}\right)$ & $102(78-148)$ & $178(118-214)$ & 0.001 \\
S. Sodium & $125(118-130)$ & $139(135.5-143)$ & 0.001 \\
S. Potassium & $4.4(3.8-4.8)$ & $4.1(3.8-4.8)$ & 0.142 \\
S.bil & $22.7(18.8-26.4)$ & $11.8(10.8-13.7)$ & 0.001 \\
S. Creat & $1.2(0.8-1.3)$ & $0.8(0.6-1.1)$ & 0.076 \\
S.albumin & $2.4(2.2-2.6)$ & $2.9(2.6-3.0)$ & 0.001 \\
INR & $2.6(2.1-2.9)$ & $1.6(1.6-1.9)$ & 0.001 \\
\hline
\end{tabular}

Table 4 Comparison of prognostic scores in patients with acute-on-chronic liver failure (ACLF) in predicting short-term Mortality

\begin{tabular}{llllll}
\hline Scores & AUROC $(95 \%$ CI) & Sensitivity(\%) & Specificity(\%) & PPV(\%) & NPV(\%) \\
\hline APACHE II & $0.94(0.889-0.987)$ & 92.7 & 86.7 & 89.5 & 90.7 \\
SOFA & $0.78(0.694-0.87)$ & 85.5 & 53.3 & 69.1 & 75 \\
MELD & $0.78(0.69-0.869)$ & 90.9 & 51.1 & 69.4 & 82.1 \\
CTP & $0.67(0.561-0.778)$ & 81.8 & 51.2 & 67.2 & 69.7 \\
\hline
\end{tabular}




\section{Discussion}

Patients with ACLF usually present with multi-organ failure with poor overall survival. There was a short term mortality of $55 \%$ in our study. This is similar to the data published from various studies worldwide. ${ }^{3,6,7}$

Alcohol was the most common cause of underlying cirrhosis in our study (in 59\%) with alcoholic hepatitis as the most common acute precipitating event (in 43\%). Hepatitis B as an etiological factor of CLD in our study is less. In the Relief trial done in European countries, the etiology of liver disease was alcohol in $78 \%$ of the patients. Similar results were shown in a study by Karavellas et al. ${ }^{9}$

In our study, a total of 35patients had type I ACLF (non hepatic insult), 65 had type II (hepatic insult). Considering these etiologies under infectious and non infectious causes as classified by APASL working committee, in our study, infectious causes amounted to $41 \%$ of the causes and non-infectious causes to $58 \%$. Similar results are shown by few recent studies done in India on patients who strictly meet the criteria of ACLF according to APASL definition. Duseja et al. ${ }^{5}$ concluded that Non hepatic insults are common acute precipitants in patients with Acute on Chronic Liver failure (ACLF). Out of 100 patients studied, 62patients had non hepatic cause, mostly SBP as a precipitant of Jalan et al. ${ }^{10}$ concluded that non hepatic insults like gastrointestinal bleeding or sepsis may start a cascade of events that culminate in end-organ dysfunction and liver failure in a patient with stable cirrhosis. They postulated the cytokine burst and profound disturbance in systemic hemodynamics and their interplay as the major pathogenesis in these patients. ${ }^{10}$

The early short term mortality was higher in patients who had UGI bleed \& sepsis. These clinical observations are supported by the fact that patients with sepsis \& SBP have defects in neutrophil functions in the form of defective neutrophil locomotion, chemotaxis, phagocytosis and bactericidal capacity. ${ }^{11,12}$

Our study shows the superiority of APACHE II in relation to other scores for predicting short-term mortality. In critically ill cirrhotic patients admitted to an ICU, some studies have demonstrated that APACHE II and SOFA performed better than CTP in predicting inhospital mortality. ${ }^{13,14}$ In a study done by Zauner et al. ${ }^{15}$ APACHE III was found to be the most reliable scoring system to separate survivors and non survivors. Yu et al. ${ }^{16}$ in concluded that the APACHE III scoring system is superior to CTP and MELD scoring systems for prognosticating in-hospital mortality among decompensated cirrhotic patients.

Limited studies have been published on the usefulness of prognostic scores in patients defined strictly as ACLF. Garg et al. ${ }^{6}$ studied the predictors of mortality in patients of ACLF. The CTP had AUROC of 0.667 whilst all three remaining scores MELD, SOFA and APACHE II had AUROCs of $>0.8$ which was significantly higher $(p<0.05)$ than that of CTP. Krishna et al. ${ }^{17}$ concluded that MELD scoring system was a better predictor of 3-month outcome than CTP score. Duseja et al. ${ }^{7}$ assessed the performance of various prognostic scores in predicting short-term mortality in patients with ACLF. They concluded that short-term mortality is high in patients with ACLF. Overall, the area under the receiver operating characteristic curve of APACHE II score (0.74) was higher than that of SOFA (0.65), MELD (0.67) and CTP score $(0.61){ }^{7}$

The CTP scoring system has been widely used to risk stratify cirrhotic patients and assess the efficiency of various therapeutic procedures, but is not ideal tool for predicting mortality or resource utilization in cirrhotic patients with multiple organ failure due to failure to assess extrahepatic prognostic factors such as cardiovascular, renal and pulmonary functions. APACHE II accurately risk stratifies critically ill patients with cirrhosis because it explains many of the factors known to influence prognosis. APACHE II incorporates parameters that measures the pulmonary, cardiac, electrolyte, acidbase and neurologic status of the patient. Hence the ultimate prognosis of patients with ACLF is significantly influenced by the degree of acute physiological derangements reflected by the acute physiology score of the APACHE model. ${ }^{17}$

There is scarcity of data on liver transplant in ACLF. Orthotropic liver transplantation remains the only definitive therapy for patients who do not improve with supportive measures to sustain life. Developing effective methods of liver support or other alternatives for transplantation and better prognostic scoring systems remains key goals to further improve overall survival rates for the condition. ${ }^{18,19}$ Liver transplantation should be performed according to prognostic scores suggesting death within the next 3 months. It should be considered earlier if HRS develops. ${ }^{1}$ Long-term results from chronic hepatitis-B-related liver disease are satisfactory. ${ }^{18,19}$

Given the limited treatment and limited medical resources, physicians require a reliable tool to risk stratify and monitor patients of ACLF. The use of various prognostic scores in patients with ACLF may help in categorizing patients to limited ICU resources and evolving therapies. So the early mortality can be predicted by applying APACHE II scoring system.

\section{Acknowledgements}

None

\section{Conflict of interest}

Author declares that there is no conflict of interest.

\section{References}

1. Sarin SK, Kumar A, Almeida JA, et al. Acute-on chronic liver failure: consensus recommendations of the Asian Pacific Association for the Study of the liver (APASL). Hepatol Int. 2009;3(1):269-282.

2. Katoonizadeh A, Laleman W, Verslype C, et al. Early features of acuteon-chronic alcoholic liver failure: a prospective cohort study. Gut. 2010;59(11):1561-1569.

3. Moreau R, Jalan R, Glines P. Acute on chronic liver failure is a distinct syndrome that develops in patients with acute decompensation of cirrhosis. Gastroenterology. 2013;144(7):1426-1437.

4. O'Shea RS, Dasarathy S, McCullough AJ. Alcoholic Liver disease. Hepatology. 2010;51(1):307-328.

5. Duseja A, Chawla YK, Dhiman RK, et al. Non-hepatic insults are common acute precipitants in patients with Acute on Chronic Liver failure (ACLF). J Dig Dis. 2010;55(11):3188-3192.

6. Garg H, Kumar A, Garg V, et al. Clinical profile and predictors of mortality in patients of acute-on-chronic failure. Dig \& Liv Dis. 2012;44(2):166-171.

7. Duseja A, Choudhary NS, Gupta S, et al. APACHE II score is superior to SOFA, CTP and MELD in predicting the short term mortality in patients with acute-on-chronic liver failure (ACLF). J Dig Dis. 2013;14(9):484-490.

8. Banares R, Nevens F, Larsen FS. Extra corporeal albumin dialysis with the molecular adsorbent recirculating system in acute on chronic liver failure: the RELIEF trial. Hepatology. 2013;57(3):1153-1162. 
9. Karvellas CJ, Pink F, McPhail M, et al. Bacteremia acute physiology and chronic health evaluation II and modified end stage liver disease are independent predictors of mortality in critically ill nontransplanted patients with acute on chronic liver failure. Crit Care Med. 2010;38(1):121-126.

10. Jalan R, Williams R. Acute-on chronic liver failure; pathophysiological basis of therapeutic options. Blood Purif. 2002;20(3):252-261.

11. Mookerjee RP, Stadlbauer U, Vidder S, et al. Neutrophil dysfunction in alcoholic hepatitis superimposed on cirrhosis is reversible \& predicts the outcome. Hepatology. 2007;46(3):831-840.

12. Li LJ, Yang Q, Huang JR, et al. Effect of artificial liver support system on patients with severe viral hepatitis: a study of four hundred cases. World J Gastroenterol. 2004;10(20):2984-2988.

13. Butt AK, Khan AA, Alam A, et al. Predicting hospital mortality in cirrhotic patients : comparison of Child-Pugh and acute physiology, age \& chronic health evaluation (APACHE III) scoring systems. Am J Gastroenterol. 1998;93(12):2469-2475.

14. Chen YC, Tian YC, Liu NJ. Prospective cohort study comparing sequential organ failure assessment and acute physiology ,age, chronic health evaluation III scoring systems for hospital mortality prediction in critically ill patients. Int J Clin Pract. 2006;60(2):160-166.
15. Zauner CA, Apsner RC, Kranz A. Outcome predictors for patients with cirrhosis of the liver in a medical ICU: a comparison of the APACHE scores \& liver specific scoring systems. Intensive care Med. 1996;22(6):559-563.

16. Yu II, Abola L. Predicting prognosis among cirrhotic patients: Child Pugh versus APACHE III versus MELD scoring systems. Phil J Gastroenterol. 2006;2:19-24.

17. Krishna RY, Saraswat VA, Das K, et al. Clinical features and predictors of outcome in acute Hepatitis A and Hepatitis E virus hepatitis on cirrhosis. Liver Int. 2009;38:392-398.

18. Starkel P, Horsmans Y, Geubel A, et al. Favourable outcome of orthotopic liver transplantation in a patient with sub-acute liver failure due to the emergence of a hepatitis B YMDD escape mutant virus. $J$ Hepatol. 2001;35(5):679-681.

19. Wai CT, Da Costa M, Sutedja D, et al. Long term results of liver transplant in patients with chronic viral hepatitis- related liver disease in Singapore. Singapore Med J. 2006;47(7):588-591. 